\title{
Discovery of an optical bow-shock around pulsar B0740-28^
}

\author{
D. H. Jones ${ }^{1,2}$, B. W. Stappers ${ }^{3,4}$, and B. M. Gaensler ${ }^{5}$ \\ 1 European Southern Observatory, Casilla 19001, Santiago 19, Chile, e-mail: hjones@eso.org \\ 2 Observatorio Cerro Calán, Departamento de Astronomía, Universidad de Chile, Casilla 36-D, Santiago, Chile \\ 3 Stichting ASTRON, 7990 Dwingeloo, The Netherlands \\ e-mail: stappers@astron.nl \\ 4 Sterrenkundig Instituut "Anton Pannekoek", 1098 SJ Amsterdam, The Netherlands \\ 5 Harvard-Smithsonian Center for Astrophysics, 60 Garden Street, Cambridge MA 02138, USA
}

Received 6 February 2002 / Accepted 24 April 2002

\begin{abstract}
We report the discovery of a faint $\mathrm{H} \alpha$ pulsar wind nebula (PWN) powered by the radio pulsar B0740-28. The characteristic bow-shock morphology of the PWN implies a direction of motion consistent with the previously measured velocity vector for the pulsar. The PWN has a flux density more than an order of magnitude lower than for the PWNe seen around other pulsars, but, for a distance $2 \mathrm{kpc}$, it is consistent with propagation through a medium of atomic density $n_{\mathrm{H}} \sim 0.25 \mathrm{~cm}^{-3}$, and neutral fraction of $1 \%$. The morphology of the PWN in the area close to the pulsar is distinct from that in downstream regions, as is also seen for the PWN powered by PSR B2224+65. In particular, the PWN associated with PSR B0740-28 appears to close at its rear, suggesting that the pulsar has recently passed through a transition from low density to high density ambient gas. The faintness of this source underscores that deep searches are needed to find further examples of optical pulsar nebulae.
\end{abstract}

Key words. ISM: general - pulsars: individual: B0740-28

\section{Introduction}

Pulsar wind nebulae (PWN) around high-velocity pulsars provide a primary insight into the interaction between a pulsar and its environment. Specifically, optical observations of such nebulae provide important information on pulsar velocities, and on the density, temperature and composition of the ambient medium. However only three pulsars are known to power optical bow-shock PWNe: B2224+65 (the "Guitar Nebula", Cordes et al. 1993), and the two millisecond pulsars (MSPs) B1957+20 (Kulkarni \& Hester 1988) and J0437-4715 (Bell et al. 1995). All three of these pulsars have high spin-down luminosities and/or high space velocities. However, these pulsars differ markedly in their spin-periods, ages and magnetic field strengths, highlighting the variety of pulsar winds which can be probed by these sources. The nebula associated with the neutron star RX J1856.5-3754 further exemplifies the variety of neutron stars known to power such nebulae (van Kerkwijk \& Kulkarni 2001). We have therefore initiated a search for optical bow-shocks around other

Send offprint requests to: D. H. Jones, e-mail: hjones@eso.org

* Based on observations made with ESO Telescopes at the La Silla Observatory (Programme 66.D-0046) and under the AAT Service Mode Programme.
Table 1. Properties of PSR B0740-28.

\begin{tabular}{lcr}
\hline \hline Spin Period $^{a}$ & $P(\mathrm{~s})$ & 0.1667 \\
Period Derivative $^{a}$ & $\dot{P}$ & $1.68 \times 10^{-16}$ \\
Spin-down Luminosity $^{a}$ & $\dot{E}\left(\mathrm{ergs} \mathrm{s}^{-1}\right)$ & $1.4 \times 10^{35}$ \\
Dispersion Measure Distance $^{b}$ & $d_{\mathrm{DM}}(\mathrm{kpc})$ & $1.9_{-0.3}^{+0.4}$ \\
Hi Distance $^{c}$ & $d_{\mathrm{HI}}(\mathrm{kpc})$ & $1.4-7.7$ \\
Proper Motion $^{d}$ & $\mu\left(\right.$ mas yr $\left.^{-1}\right)$ & $29 \pm 1$ \\
Position Angle $^{d}$ & $\theta\left(^{\circ}\right)$ & $269 \pm 1$ \\
\hline
\end{tabular}

${ }^{a}$ Arzoumanian et al. (1994). ${ }^{b}$ Taylor \& Cordes (1993).

${ }^{c}$ Koribalski et al. (1995). ${ }^{d}$ Bailes et al. (1990).

pulsars in order to characterise the properties of the associated pulsars, pulsar winds and ambient environments.

During the first two nights of this programme (January 4 and 5, 2001), we discovered an optical bow-shock nebula around the radio pulsar B0740-28. Discovered at Bologna (Salter 1970), this pulsar was subsequently shown to be rapidly spinning down (McCulloch et al. 1973). Its correspondingly high spin-down luminosity, $\dot{E}=4 \pi I \dot{P} / P^{3}$, (where $I=10^{45} I_{45} \mathrm{~g} \mathrm{~cm}^{2}$ is the pulsar moment of inertia; see Table 1), but likely moderate distance and high transverse velocity make it a promising target for powering an observable $\mathrm{H} \alpha$ nebula. We note that the maximum distance derived from $\mathrm{H}$ I 
Table 2. Log of observations for the PSR B0740-28 field.

\begin{tabular}{lcc}
\hline \hline Date & 4, 5 January 2001 & 14 April 2001 \\
Instrument & SUSI-2/NTT & TTF $^{a} /$ AAT \\
Pixel Scale $\left("\right.$ pix $\left.^{-1}\right)$ & 0.161 & 0.373 \\
Filter $^{b}$ (nm) & $656 / 7$ & $656 / 2$ \\
Exposure Time (s) & 6480 & 2700 \\
Transparency & photometric & photometric \\
Mean Seeing (") & $\sim 0.9$ & $\sim 1.4$ \\
Continuum Frame & None & $600 \mathrm{~s}$ in $R 1^{c}$ \\
\hline
\end{tabular}

${ }^{a}$ Using the blue Fabry-Perot and $R 0(668 / 21)$ filter.

${ }^{b}$ Central wavelength and FWHM in nanometers.

${ }^{c}$ TTF $R 1$ filter $(707 / 26)$.

measurements given in Table 1 is large, but in Sect. 5 we argue for a distance closer to that derived from the dispersion measure. See Chatterjee \& Cordes (2002) for a recent review.

\section{Observations and reduction}

The nebula around PSR B0740-28 was discovered in narrowband frames taken through the $656 / 7 \mathrm{~nm}$ filter of SUSI-2 at the $3.5 \mathrm{~m}$ New Technology Telescope (NTT), La Silla (Fig. 1a). A separate continuum-subtracted narrowband frame (Fig. 1b) was derived from subsequent imaging with the Taurus Tunable Filter (TTF; BlandHawthorn \& Jones 1998) at the $3.9 \mathrm{~m}$ Anglo-Australian Telescope (AAT). Figure 1c shows the NTT image in Fig. 1a smoothed with a $5 \times 5$-pixel boxcar kernel. The observations are summarised in Table 2. Raw frames were treated using standard techniques for bias and flat-field correction, image registration and co-addition. CCD defects and cosmic rays were removed using cross-pixel interpolation on the final frame, in order to preserve as much of the faint nebula signal as possible.

An astrometric solution was derived for the higherresolution NTT image using the IRAF task ccmap. A Legendre polynomial with 11 coefficients was found to give the best fit in $x$ and $y$ to 24 USNO Catalog reference stars across the field. The fitting option used in CCMAP sees a linear term (with coefficients dependent on translation, scaling, rotation and skew), computed separately from a distortion term, which is a polynomial fit to the residuals of the linear term. No distortion residuals were evident in a test transformation of the USNO star coordinates to those of the image. The rms scatter was $(\Delta \alpha, \Delta \delta)=\left( \pm 0.028^{\prime \prime}, \pm 0.038^{\prime \prime}\right)$ and uniform across the field.

The location of PSR B0740-28 was determined by applying the proper motion determined by Bailes et al. (1990) to the position measured by Fomalont et al. (1992). This places the pulsar at $(\alpha, \delta)=$ $(07: 42: 49.041,-28: 22: 44.02)(\mathrm{J} 2000)$ at the mean time of observation, 1 !' 3 inside the leading edge of the nebula on its long axis (Fig. 1). It is not coincident with the prominent star near the head of the nebula.
Bailes et al. (1990) determine a proper motion position angle (measured through east) of $\theta=269 \pm 1^{\circ}$, i.e. due west. This is consistent with the direction of the motion implied by the symmetry axis of the nebula and confirms the association.

\section{Flux and morphology}

The optical emission from pulsar wind nebulae is purely Balmer. The $\mathrm{H} \alpha$ flux density in the NTT image was calibrated using observations of the planetary nebula flux standards 235.3-03.9 and 278.6-06.7 (Dopita \& Hua 1997). Atmospheric extinction was estimated using the CTIO measurements of Stone \& Baldwin (1983). For the planetary nebula standards, the $656 / 7$ filter admits flux from the adjacent [N II] $\lambda 6548$ and [N II] $\lambda 6584$ lines, in addition to $\mathrm{H} \alpha$. The [N II] contribution for 235.3-03.9 (37\%) is more significant than $278.6-06.7$ (15\%), although the $656 / 7$ fluxes were corrected for it in both cases.

The H $\alpha$ flux over the entire PSR B0740-28 nebula is $5 \times 10^{-5}$ photons $\mathrm{s}^{-1} \mathrm{~cm}^{-2}$, around two orders of magnitude fainter than the nebulae powered by J0437-4715 (Bell et al. 1995) and B1957+20 (Kulkarni \& Hester 1988). The $\mathrm{H} \alpha$ images in Fig. 1 show a distinctive key-hole shape which can be divided into three main regions. The most western component, or "head", has an almost circular shape, with the pulsar located at the leading edge. Approximately $20^{\prime \prime}$ from the front of the shock, the nebula broadens into a fan-shaped tail and a bridge of emission connecting the two sides of the nebula can be seen. At the eastern limit of emission, some $45^{\prime \prime}$ from the apex, the nebula appears to show a boundary with opposite concavity to that of the apex, closing the tail. Figure $1 \mathrm{~b}$ shows no emission within the interior of the head although deeper images are needed to confirm this.

Variations in the surface brightness across the extent of the nebula are seen in Figs. 1a and 1b; see Table 3 for a summary. The knot at Region A, located at the juncture of the "fan" and "head" section, and its southern counterpart both have surface brightness greater than that of the overall nebula.

\section{Nebular geometry}

When a star moves at a supersonic velocity with respect to the interstellar medium (ISM), a bow-shock forms at the interface between the stellar wind and the ambient medium. For pulsars the wind is relativistic. Assuming an isotropic wind, the stand-off distance, $r_{\mathrm{w}}$, separating the pulsar from the contact discontinuity of the shock, can be derived from the balance between ram pressure and the outflowing wind pressure,

$r_{\mathrm{w}}=\left(\frac{\dot{E}}{4 \pi c \rho_{0} v_{\mathrm{p}}^{2}}\right)^{1 / 2} \mathrm{~cm}$

where $\rho_{0}=2.2 \times 10^{-24} n_{\mathrm{H}} \mathrm{g} \mathrm{cm}^{-3}$ is the ISM density, $n_{\mathrm{H}}$ is the hydrogen number density and $v_{\mathrm{p}}\left(\mathrm{cm} \mathrm{s}^{-1}\right)$ is the space velocity of the pulsar. 

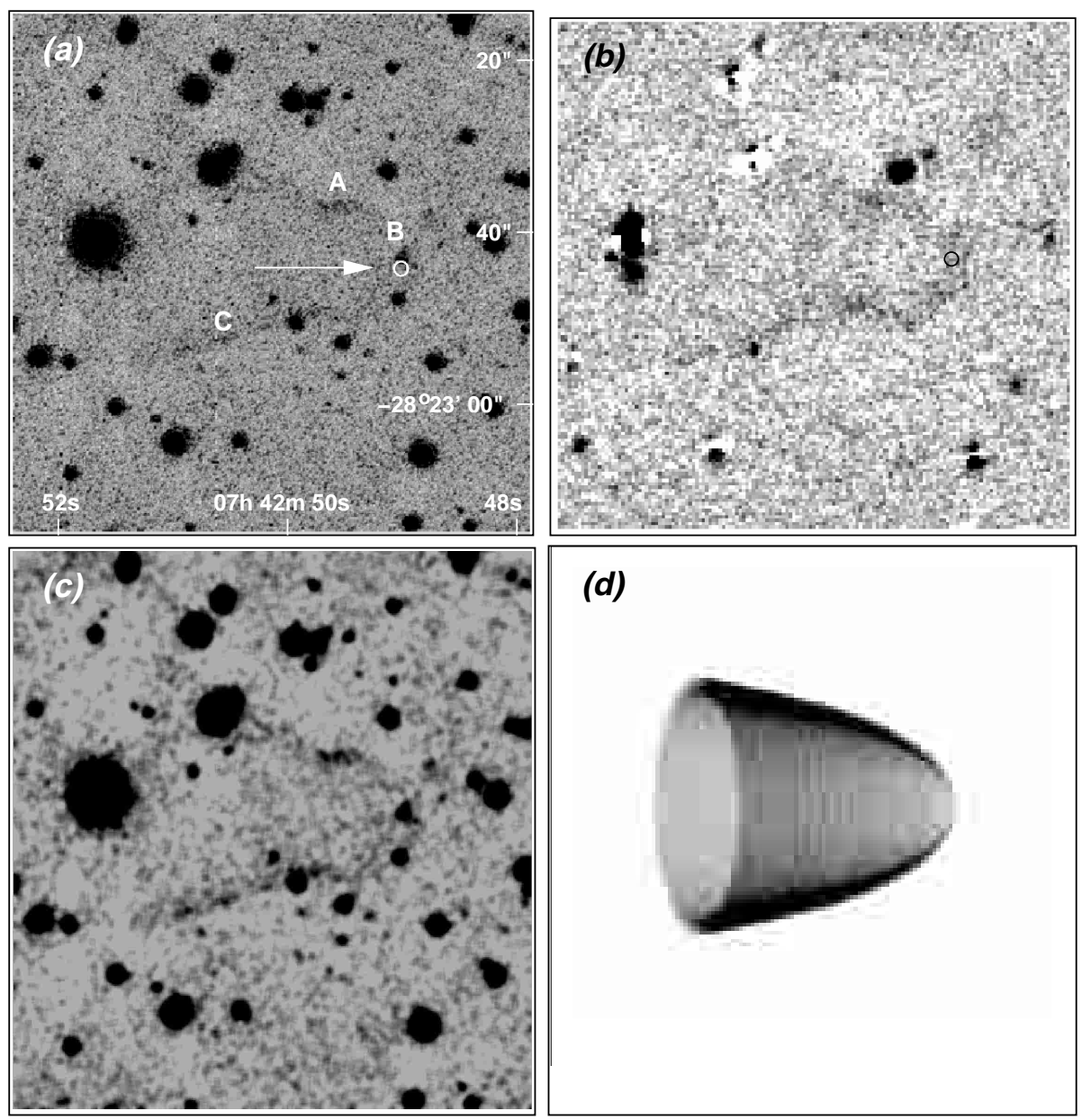

(d)

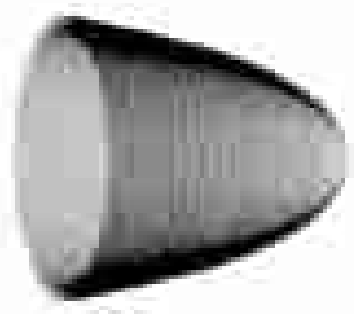

Fig. 1. a) SUSI-2/NTT discovery image ( $\mathrm{H} \alpha+$ continuum) of the nebula associated with PSR B0740-28. The arrow indicates the direction and distance travelled by the pulsar over 500 yrs. Coordinate epoch is J2000. b) Continuum-subtracted H $\alpha$ image of the same field taken with TTF on the AAT. Some residuals due to saturated stars and in-focus ghost images are present. c) Image a) smoothed using a $5 \times 5$-pixel boxcar kernel. Panels a)-c) are 1 arcmin on a side with north up, east left, with the pulsar position indicated by a circle. d) Illustrative example of a simple surface of rotation based on Eq. (3), that assumes the pulsar is moving $20^{\circ}$ to the plane of the sky.

The shock apex was determined by finding the peak emission lying along the pulsar proper motion direction and is located 1 1". 3 from the pulsar. However this does not correspond to $r_{\mathrm{w}}$, as the Balmer emission comes from a thin shell upstream from the contact discontinuity. Aldcroft et al. (1992) find for B1957+20 that the measured pulsar-apex separation is $30 \%$ larger than $r_{\mathrm{w}}$. Hence we adopt $r_{\mathrm{w}}=1^{\prime \prime} 0=0.005 d_{\mathrm{kpc}} \mathrm{pc}$, where $d_{\mathrm{kpc}}$ is the pulsar distance in kpc. The proper motion, when corrected for galactic rotation, corresponds to a projected space velocity of $v_{\mathrm{p}}=1.3 \times 10^{7} d_{\mathrm{kpc}} / \cos i \mathrm{~cm} \mathrm{~s}^{-1}$, where $i$ is the angle of this velocity vector to the plane of the sky. Substituting $v_{\mathrm{p}}$ into Eq. (1) and rearranging gives:

$n_{\mathrm{H}}=\frac{4 \cos ^{4} i I_{45}}{d_{\mathrm{kpc}}^{4}} \mathrm{~cm}^{-3}$.

Using the upper and lower limits to the distance given in Table 1 and assuming that $i$ is small then $0.002 \lesssim$ $n_{\mathrm{H}} \lesssim 1 \mathrm{~cm}^{-3}$. If we assume the dispersion measure
Table 3. Mean surface brightness of different regions.

\begin{tabular}{cc}
\hline $\begin{array}{c}\text { Region } \\
\text { (see Fig. 1a) }\end{array}$ & $\begin{array}{c}\text { Surface Brightness } \\
\left(\mathrm{erg} \mathrm{s}^{-1} \mathrm{~cm}^{-2} \operatorname{arcsec}^{-2}\right)\end{array}$ \\
\hline A & $7 \times 10^{-19}$ \\
B & $4 \times 10^{-19}$ \\
C & $5 \times 10^{-19}$ \\
entire nebula & $3 \times 10^{-19}$ \\
\hline
\end{tabular}

distance $d_{\mathrm{kpc}}=2$, (which we argue for in Sect. 5), then the density is $n_{\mathrm{H}} \sim 0.25 \mathrm{~cm}^{-3}$.

Recent derivations of the geometry of thin momentumbalance bow-shocks give a generic form for the shock that scales with stand-off distance (Chen et al. 1996; Wilkin 1996). We have compared the shape of the PSR B0740-28 nebula with the expression of Chen et al. (1996),

$\frac{x}{r_{\mathrm{w}}}+1=\frac{3}{10}\left(\frac{y}{r_{\mathrm{w}}}\right)^{2}+\left(\frac{3}{280} \frac{y}{r_{\mathrm{w}}}\right)^{4}$ 
Here, our axes lie in the plane of the sky, such that the $y$-axis lies along the proper motion direction, $x$ is perpendicular to it and the origin is located at the pulsar. The best fit to 14 locations around the "head" gives a standoff distance of $1^{\prime \prime} .2 \pm 0.1$ and proper motion position angle of $276 \pm 3^{\circ}$ which are consistent with the measured values. However some deviation from an exact fit occur on the southern edge of the shock front. Modelling by Wilkin (2000) shows that either wind asymmetry or density gradients in the ISM are able to reproduce such variations. Observations of PWNe around other pulsars have demonstrated marked anisotropies in the wind flow away from the pulsar, (Hester 1998; Gaensler et al. 2002), while large variations in the ISM density are seen in the region of the Guitar nebula (Chatterjee \& Cordes 2002).

\section{Discussion}

Cordes (1996) shows that the $\mathrm{H} \alpha$ flux density in the nebula can be expressed in terms of observables as,

$F_{\alpha}=\frac{10^{-2.47} X n_{\mathrm{H}} v_{7}^{3} \theta_{\mathrm{s}}^{2}}{\cos ^{5} i}$ photons $\mathrm{cm}^{-2} \mathrm{~s}^{-1}$,

where $X$ is the neutral fraction, $v_{7}=v_{\mathrm{p}} / 10^{7}$ and $\theta_{\mathrm{s}}$ is the observed angular distance. The $2 \mathrm{kpc}$ distance derived from the dispersion measure (Taylor \& Cordes 1993) is consistent with the amount of reddening and closed shape of the nebula, in the following ways.

The observed reddening in the direction of PSR B0740-28 is $A_{\mathrm{v}} \approx 1.5$ magnitudes at $d \approx 2 \mathrm{kpc}$ and distances beyond, corresponding to a reduction in $\mathrm{H} \alpha$ flux by a factor of $\sim 3$ (Neckel \& Klare 1980). Combining this with a neutral fraction $X=0.01$, (reasonable for a warm ionised ISM with the $n_{\mathrm{H}} \sim 0.25 \mathrm{~cm}^{-3}$ calculated earlier from Eq. (2) with $d_{\mathrm{kpc}}=2$ ), gives good agreement with the observed flux. At larger distances the density derived from Eq. (2) implies the pulsar is in the hot phase of the ISM where the neutral fraction is very small, thereby further reducing the predicted flux.

The direction of the nebula with respect to the plane of the sky will also affect the measured flux. Using the shock morphology formula given in Eq. (3), we generated a three-dimensional model of the shock and then determined its shape projected on the plane of the sky for various values of $i$. We find that to best match the observed standoff distance and opening angle $i$ must be small $\left(\lesssim 25^{\circ}\right)$ and thus its contribution to the low flux is small.

The overall morphology of the PSR B0740-28 nebula is very similar to the Guitar nebula where the "neck" and "body" regions (Cordes et al. 1993) are analogous to our "head" and "fan" regions respectively. However, there are some differences; the head is more circular than the cylindrical neck of the Guitar nebula and the fan is more rectilinear than the bulbous body of the Guitar nebula. We note, however, that both nebulae show stronger $\mathrm{H} \alpha$ emission in the transition between the narrow and broad regions. These could indicate regions of increased density on sub-parsec scales, given that the full extent of the nebula is $\sim 0.6 \mathrm{pc}$ if $2 \mathrm{kpc}$ away. Alternatively, the stronger emission may indicate the transition between different physical regimes of the nebula.

The Guitar nebula emission shows two distinct bubble regions (Cordes et al. 1993). The fainter, inner bubble is analogous to the "bridge" of emission that we see in PSR B0740-28. It is possible to generate these apparently closed structures via projection of a truly open structure. This can occur if the nebula is slightly inclined, as is shown in Fig. 1d for a cone assumed to be inclined at $20^{\circ}$.

The apparently closed nature of the PSR B0740-28 nebula suggests that in the rear region of the nebula, the ram pressure due to the pulsar motion is no longer important and we could instead be seeing the point where the pressure in the wind is balanced by the ISM pressure - it is possible that the break between the "head" and "tail" components of the PWN may correspond to the transition between these two sources of confinement. However, this explanation is not consistent with the entire PWN being embedded in gas of uniform density, since the pulsar's motion must be supersonic to form a bow shock, but in which case there can be no pressure balance at the rear of the PWN. In order to explain the observed morphology, we propose that the pulsar has recently passed through a strong density gradient in the ISM, such that the pulsar's motion is supersonic in the comparatively dense medium $\left(n_{\mathrm{H}} \sim 0.25 \mathrm{~cm}^{-3}\right)$ through which it is currently propagating, but was subsonic in low density material $\left(n_{\mathrm{H}} \lesssim 0.003 \mathrm{~cm}^{-3}\right)$ surrounding the closed end of the PWN. Similar arguments can be invoked to explain the morphology of the Guitar PWN powered by PSR B2224+65.

Considering the latter region, the requirement that the pulsar be moving slower that the local sound speed implies an upper limit to the pulsar's distance of $3-4 \mathrm{kpc}$, as no ISM component has a sound speed faster than $\sim 500 \mathrm{~km} \mathrm{~s}^{-1}$. If PSR B0740-28 is at $2 \mathrm{kpc}$, then the pulsar wind pressure, $P_{\mathrm{w}}=\dot{E} /\left(4 \pi r_{\mathrm{b}}^{2} c\right)$ approximately balances the ISM pressure, $P_{\mathrm{ISM}} \sim 5 \times 10^{-14} \mathrm{~Pa}$, at the the location of the closed region (where $r_{\mathrm{b}}$ is the distance to the back of the nebula), providing further support for the lower distance argued for above. In the interpretation we propose, the closed region of the PWN corresponds to the static or "ghost" nebulae proposed by Blandford et al. (1973). The faintness of this region is consistent with the expectation that such sources should generally never be observable (Gaensler et al. 2000); in the case of PSR B0740-28, it was only through the presence of brighter emission near the pulsar that this closed region was identified.

Bucciantini \& Bandiera (2001) have recently shown that classical thin-shock models are not appropriate for pulsar bow-shock nebulae. However, here we find reasonable agreement between the classical models and the shape of the head of the PSR B0740-28 nebula. It is likely though that the microphysical processes they discuss are responsible for at least some of the morphological characteristics of these PWNe. Indeed Bucciantini \& Bandiera (2001) point out that perhaps PSR B0740-28 is an 
intermediate case between the nebulae associated with the MSPs and the Guitar nebula. Interestingly, they also predict that, based on the ISM conditions, this nebula should be readily detectable. However, we find that it is considerably less-so than the others. Clearly, increasing the presently small sample of optical bow-shock nebulae remains a key step towards being able to generalise about the ISM conditions within the vicinity of pulsars.

Acknowledgements. We are grateful to the NTT Team and J. Bland-Hawthorn for the quality of the data obtained at the NTT and AAT respectively. We are also grateful to the referee, R. Bandiera, whose comments helped to improve the final draft. BWS is supported by NWO Spinoza grant 08-0 to E. P. J. van den Heuvel. BMG acknowledges the support of a CfA Clay Fellowship. DHJ would like to acknowledge R. A. Faulkner (1945-2001) for encouraging an early interest in astronomy.

\section{References}

Aldcroft, T. L., Romani, R. W., \& Cordes, J. M. 1992, ApJ, 400, 638

Arzoumanian, Z., Nice, D. J., Taylor, J. H., \& Thorsett, S. E. 1994, ApJ, 422, 671

Bailes, M., Manchester, R. N., Kesteven, M. J., Norris, R. P., \& Reynolds, J. E. 1990, MNRAS, 247, 322

Bell, J. F., Bailes, M., Manchester, R. N., Weisberg, J. M., \& Lyne, A. G. 1995, ApJ 440, L81
Blandford, R. D., Ostriker, J. P., Pacini, F., \& Rees, M. J. 1973, A\&A, 23, 145

Bland-Hawthorn, J., \& Jones, D. H. 1998, in Optical Astronomical Instrumentation, ed. S. D'Odorico, Proc. SPIE, 3355, 855

Bucciantini, N., \& Bandiera, R. 2001, A\&A, 375, 1032

Chatterjee, S., \& Cordes, J. 2002 [astro-ph/0201062]

Chen, Y., Bandiera, R., \& Wang, Z. 1996, ApJ, 469, 715

Cordes, J. M., Romani, R. W., \& Lundgren, S. C. 1993, Nature, 362,133

Cordes, J. M. 1996, in IAU Colloq., 160, 393

Dopita, M. A., \& Hua, C. T. 1997, ApJS, 108, 515

Fomalont, E. B., Goss, W. M., Lyne, A. G., Manchester, R. N., \& Justtanont, K. 1992, MNRAS 258, 497

Gaensler, B. M., Stappers, B. W., Frail, D. A., et al. 2000, MNRAS, 318, 58

Gaensler, B. M., Arons, J., Kaspi, V. M., et al. 2002, ApJ, 509, in press [astro-ph/0110454]

Hester, J. J. 1998, Mem. Soc. Astron. It. 69, 883

Koribalski, B., Johnston, S., Weisberg, J. M., \& Wilson, W. 1995, ApJ, 441, 756

Kulkarni, S. R., \& Hester, J. J. 1988, Nature, 335, 801

McCulloch, P. M., Komesaroff, M. M., Ables, J. G., Hamilton, P. A., \& Rankin, J. M. 1973, Astrophys. Lett. 14, 169

Neckel, Th., \& Klare, G. 1980, A\&AS, 42, 251

Salter, C. J. 1970, IAUC, 2295

Stone, R. P. S., \& Baldwin, J. A. 1983, MNRAS, 204, 347

Taylor, J. H., \& Cordes, J. M. 1993, ApJ, 411, 674

Wilkin, F. P. 1996, ApJ, 459, L31

Wilkin, F. P. 2000, ApJ, 532, 400

van Kerkwijk, M. H., \& Kulkarni, S. R. 2001, A\&A, submitted [astro-ph/0110065] 